\title{
Lesiones deportivas quirúrgicas en el Hospital Ángeles Mocel: experiencia de 235 casos
}

\author{
Surgical sports injuries at Hospital Ángeles Mocel: experience of 235 cases
}

Alejandro García-Cacho Lascurain*

Citar como: García-Cacho LA. Lesiones deportivas quirúrgicas en el Hospital Ángeles Mocel: experiencia de 235 casos. Acta Med. 2021; 19 (1): 45-51. https://dx.doi.org/10.35366/98569

\section{Resumen}

Introducción: Las lesiones deportivas se han convertido en un reto tanto para el médico general como para el especialista. Conocer e investigar las principales lesiones que requieren tratamiento quirúrgico favorece su prevención, diagnóstico y tratamiento. Material y métodos: Se realizó un estudio retrospectivo longitudinal de un periodo de cinco años en el Hospital Ángeles Mocel, basado en los datos del Servicio de Ortopedia. Resultados: Se documentaron un total de 251 cirugías. El deporte de mayor incidencia de lesiones deportivas quirúrgicas es el fútbol, con $39 \%$ para ambos géneros. Las lesiones meniscales y lesiones de ligamento cruzado anterior (LCA) son los diagnósticos con mayor frecuencia. La cirugía dominante es la artroscopia de rodilla, así como la plastia del LCA. Conclusión: Se requiere mayor número de variables en el estudio para poder realizar una conclusión más exacta $\mathrm{y}$ afirmativa.

Palabras clave: Lesiones deportivas, cirugía, futbol, lesión menisco.

\section{INTRODUCCIÓN}

El deporte es una actividad milenaria que históricamente - desde la antigua Grecia e incluso en las culturas prehispánicas, hasta nuestros días- sigue teniendo un papel importante en la vida de la sociedad. En los últimos años ha tomado un rol de gran importancia en la vida de las personas, ya sea como espectadoras o como practicantes de éste, por razones sociales, culturales y de salud. Sus beneficios se han

\section{Abstract}

Introduction: Sport injuries have become a challenge for the physician as well as specialist and to know the main injuries that require surgery, helps us with prevention, diagnostics and treatment. Material and methods: It's a retrospective longitudinal study of five years done in the Hospital Ángeles Mocel based on the data of the orthopedics service. Results: Total of 251 surgical procedures. The most common sport with sport injuries is soccer $39 \%$ in both genders, meniscus injuries and $A C L$ injuries represent the principal diagnostics. The main surgery was knee arthroscopy as well as ACL reconstruction. Conclusion: More variables are needed in order to have a more affirmative conclusion.

Keywords: Sports injuries, surgery, soccer, meniscus tear.

documentado y estudiado en diversos ámbitos, debido a su relevancia e impacto en la prevención de enfermedades crónicas. ${ }^{1}$ Derivado de lo anterior, existe un avance importante en las últimas décadas para el diagnóstico, tratamiento, recuperación y prevención de las lesiones deportivas.

Se entiende como lesión deportiva cualquier tipo de dolor o daño físico que se produce como resultado de realizar deporte, actividad física o ejercicio. ${ }^{2}$ Estas lesiones abarcan todas las edades y grupos etarios. Además, se pue-

\footnotetext{
* Ortopedia y Traumatología. Facultad Mexicana de Medicina de la Universidad La Salle México. Hospital Ángeles Mocel. Ciudad de México, México.
}

\section{Correspondencia:}

Alejandro García Cacho Lascuráin

Correo electrónico: agarciacacho@gmail.com

Aceptado: 18-05-2020.

www.medigraphic.com/actamedica

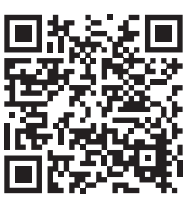


den dividir en lesiones agudas (producidas por contacto o autoagresión), accidentales o crónicas por sobrecarga. ${ }^{3}$ Las lesiones deportivas se caracterizan por ser lesiones musculoesqueléticas. De acuerdo con el Instituto Belisario Domínguez, una de cada 17 personas que realizan deporte presenta algún tipo de lesión deportiva. ${ }^{4}$

El presente artículo analiza las principales lesiones deportivas que fueron atendidas quirúrgicamente en el Hospital Ángeles Mocel y los deportes más comunes que provocan las lesiones deportivas. La importancia de conocer esta información radica en generar diagnósticos más certeros en el ámbito del deporte, así como un tratamiento individualizado y especializado, sumado a una prevención más efectiva.

\section{MATERIAL Y MÉTODOS}

El presente estudio es de tipo retrospectivo y longitudinal, con datos del expediente del archivo clínico del Hospital Ángeles Mocel de la Ciudad de México, y fueron obtenidos a través del Servicio de Ortopedia y Traumatología del periodo de julio de 2013 a julio de 2018. Se incluyeron 255 pacientes de ocho a 67 años que presentaron una lesión aguda a consecuencia de alguna actividad física o deportiva y cuyos expedientes fueron ingresados al Servicio de Ortopedia y Traumatología. Se utilizó una extracción de datos estandarizada para recabar la información. Posteriormente, los datos fueron categorizados según el deporte o actividad deportiva similar, el diagnóstico clínico e imagenológico y por el tratamiento quirúrgico aplicado. La base de datos contiene edad, género, diagnósticos y tratamiento realizado. Los criterios de exclusión fueron las lesiones musculoesqueléticas, cuyo mecanismo de lesión no ocurrió durante la realización de actividad deportiva, las lesiones deportivas crónicas, las lesiones craneoencefálicas o neurológicas, del tórax o abdominales, los mecanismos de lesión desconocido y las lesiones por accidentes automovilísticos y motocicleta. Del total de pacientes 20 (7.8\%) fueron ingresados al hospital para un tratamiento conservador exclusivamente, por lo que no se incluyen para los fines de esta investigación. Se realizó análisis estadístico de las variables, empleando estadística descriptiva, se usaron medidas de tendencia central de acuerdo con el tipo de variable. Además, se realizó la prueba de $\chi^{2}$ para comparar las variables entre los géneros y el tipo de lesión, mecanismo de lesión, considerándose significativo cuando el valor de $p$ fue menor de $<0.05$.

Los criterios de inclusión utilizados en el presente estudio abarcaron a aquellos pacientes que presentaban una lesión deportiva y que, por consecuencia, fueron sometidos a tratamiento quirúrgico. El diagnóstico, por ende, se realizó con los datos clínicos propios de cada patología, estudios de gabinete, tales como radiografías, tomografías y resonancias magnéticas, que se llevaron a cabo por el personal adscrito al Servicio de Ortopedia con apoyo del Servicio de Radiología. Para esta investigación, se tomaron en cuenta la edad, sexo, diagnóstico de lesión por práctica deportiva, localización y tratamiento realizado por algún médico ortopedista adscrito al Hospital Ángeles Mocel.

\section{RESULTADOS}

De un total de 255 pacientes, a 235 (92.1\%) se les realizó algún procedimiento quirúrgico y sólo a 20 (7.8\%) un tratamiento conservador. La media de edad fue de 33.5 años, mediana de 33 años, DE 12.4, con un máximo de 67 y mínimo de ocho años. En cuanto al género, se estudiaron a $200(85 \%)$ hombres y a $35(15 \%)$ mujeres. La estancia intrahospitalaria reportó un promedio de 1.7 días, mediana de dos días, DE 1.5.

Para el estudio se consideraron un total de 20 deportes, mismos que se presentan en la Tabla 1, categorizados por actividad deportiva y número de pacientes. Se puede observar que el deporte que presentó mayores lesiones deportivas fue el fútbol, con un total de 94 pacientes (41.7\%), seguido de la bicicleta con 32 pacientes (14.2\%) (Figura 1).

Se tomaron en cuenta 19 diferentes categorías de diagnósticos (Tabla 1), mismos que suman 308 y se desglosan por el número de pacientes atendidos y su porcentaje. Los principales diagnósticos quirúrgicos fueron (en orden de presentación): lesión de menisco reportada en 82 casos (27.1\%); lesión de ligamento cruzado anterior (grado II y III) en 59 casos (19.5\%) y tendón de Aquiles parcial o total en 28 casos (9.2\%). En la figura 2 se puede observar el comparativo de diagnósticos de mayor a menor.

Se realizaron un total de 251 cirugías, mismas que se agruparon en 18 categorías (Tabla 1). La cirugía más frecuente fue artroscopia de rodilla con 64 intervenciones (25.5\%); plastia del ligamento cruzado anterior (LCA) por vía artroscópica en 51 cirugías (20.3\%), plastia del tendón de Aquiles, abierta y percutánea, en 31 cirugías (12.4\%) (Figura 3).

Se realizó relación del mecanismo de lesión por género, la cual reportó en el género masculino (por orden de presentación): fútbol en 87 pacientes (43.5\%); ciclismo 28 pacientes (14\%); básquetbol 16 pacientes (8\%); carrera 12 pacientes (6\%); fútbol americano en 11 pacientes (5.5\%). En el género femenino (por orden de presentación): fútbol en siete pacientes $(20 \%)$; carrera en seis pacientes $(17.1 \%)$; ciclismo en cuatro pacientes (11.4\%), baile en cuatro pacientes (11.4\%), esquí, fútbol americano y patinaje con dos casos (5.7\%), respectivamente. La relación por género y mecanismo de lesión reportó valores significativos con $\mathrm{p} \leq 0.005$.

De igual modo, se correlacionó el tipo de lesión producido por género, se reportó en los pacientes masculinos 
Tabla 1: Deportes, diagnóstico y cirugías.

\begin{tabular}{|c|c|c|c|c|c|c|c|c|}
\hline Deporte & Pacientes & $\%$ & Categoría & Diagnóstico & $\%$ & Cirugías & $\mathrm{n}$ & $\%$ \\
\hline Fútbol & 94 & 41.7 & $\begin{array}{l}\text { Lesión de } \\
\text { menisco }\end{array}$ & 82 & 27.1 & $\begin{array}{l}\text { Artroscopia de } \\
\text { rodilla }\end{array}$ & 64 & 25.5 \\
\hline Bicicleta & 32 & 14.2 & Lesión del LCA & 59 & 19.5 & $\begin{array}{l}\text { Plastia del LCA } \\
\text { artroscópica }\end{array}$ & 51 & 20.3 \\
\hline Carrera & 18 & 8.0 & Tendón de Aquiles & 28 & 9.2 & $\begin{array}{l}\text { Tendón de } \\
\text { Aquiles }\end{array}$ & 31 & 12.4 \\
\hline Básquetbol & 18 & 8.0 & $\begin{array}{l}\text { Fractura de mano/ } \\
\text { muñeca }\end{array}$ & 22 & 7.3 & RAFI clavícula & 12 & 4.8 \\
\hline $\begin{array}{l}\text { Fútbol } \\
\text { americano }\end{array}$ & 13 & 5.7 & $\begin{array}{l}\text { Fractura de } \\
\text { clavícula }\end{array}$ & 14 & 4.6 & RCFP & 11 & 4.4 \\
\hline Pesas & 6 & 2.6 & Lesión condral & 14 & 4.6 & $\begin{array}{l}\text { RAFI mano/ } \\
\text { muñeca }\end{array}$ & 11 & 4.4 \\
\hline $\begin{array}{l}\text { Artes } \\
\text { marciales }\end{array}$ & 6 & 2.6 & Lesión muscular & 12 & 3.9 & Otros & 11 & 4.4 \\
\hline Baile & 5 & 2.2 & Otra & 10 & 3.3 & $\begin{array}{l}\text { RAFI acromio- } \\
\text { clavicular }\end{array}$ & 11 & 4.4 \\
\hline $\begin{array}{l}\text { Esquí } \\
\text { nieve }\end{array}$ & 5 & 2.2 & $\begin{array}{c}\text { Luxación } \\
\text { acromioclavicular }\end{array}$ & 10 & 3.3 & $\begin{array}{c}\text { Desbridamiento/ } \\
\text { drenaje }\end{array}$ & 10 & 4.0 \\
\hline Atletismo & 4 & 1.7 & Fractura de tobillo & 8 & 2.6 & RAFI tobillo & 8 & 3.2 \\
\hline Equitación & 3 & 1.3 & $\begin{array}{c}\text { Manguito rotador/ } \\
\text { SLAP }\end{array}$ & 7 & 2.3 & $\begin{array}{l}\text { Artroscopia de } \\
\text { hombro }\end{array}$ & 7 & 2.8 \\
\hline Vóleibol & 3 & 1.3 & $\begin{array}{l}\text { Fractura de codo/ } \\
\text { hombro }\end{array}$ & 7 & 2.3 & $\begin{array}{l}\text { Reparación } \\
\text { muscular }\end{array}$ & 5 & 2.0 \\
\hline Patinaje & 2 & 0.9 & Fractura de tibia & 6 & 2.0 & RAFI tibia & 5 & 2.0 \\
\hline Beisbol & 2 & 0.9 & $\begin{array}{c}\text { Lesión } \\
\text { ligamentaria }\end{array}$ & 6 & 2.0 & Plastia rotuliano & 4 & 1.6 \\
\hline Crossfit & 2 & 0.9 & Fractura de pie & 4 & 1.3 & RAFI pie & 3 & 1.2 \\
\hline Tenis & 2 & 0.9 & $\begin{array}{l}\text { Fractura de } \\
\text { antebrazo }\end{array}$ & 4 & 1.3 & RAFI codo & 3 & 1.2 \\
\hline Surf & 2 & 0.9 & Tendón rotuliano & 4 & 1.3 & Columna & 2 & 0.8 \\
\hline Patineta & 1 & 0.4 & $\begin{array}{c}\text { Fractura de } \\
\text { vértebra }\end{array}$ & 3 & 1.0 & Húmero & 2 & 0.8 \\
\hline Golf & 1 & 0.4 & Hernia disco & 2 & 0.6 & & & \\
\hline Paracaídas & 1 & 0.4 & & & & & & \\
\hline Total & 225 & 100.0 & & 302 & 100.0 & & 251 & 100.0 \\
\hline
\end{tabular}

(por orden de presentación): lesión de menisco en 61 casos (30\%); lesión del LCA en 42 casos (21\%); tendón de Aquiles en 29 casos (14.5\%); fractura de mano/muñeca en 15 casos (7.5\%); fractura de clavícula en 14 casos (7\%); lesión muscular 13 casos (6.5\%); lesión condral 11 casos (5.5\%); luxación acromioclavicular 10 casos (5\%); manguito rotador, lesión labrum anterior a posterior (SLAP por su siglas en inglés) y fractura de tobillo en ocho casos (4\%), respectivamente. En el género femenino, por orden de presentación: lesión de menisco en 19 casos (54.3\%); lesión del LCA en 14 casos (40\%); lesión condral en cinco casos (14.3\%); fractura de mano/muñeca y lesión del tendón de Aquiles en tres casos (8.6\%), respectivamente. No se reportó significancia estadística en correlación al diagnóstico y género.

Se correlacionó el tipo de lesión por el mecanismo (actividad realizada), registramos sólo los deportes con una población $\geq 10$, en orden de presentación: fútbol (94 pacientes), lesión del LCA en $29.8 \%$, lesión de menisco en $27.7 \%$, lesión del tendón de Aquiles en 14.9\%; ciclismo 
(32 pacientes) lesión de mano/muñeca en 34.4\%, lesión de brazo/antebrazo en 34.4\%; carrera (18 pacientes) lesión de menisco en 33.3\%, lesión del tendón de Aquiles en 33.3\%; básquetbol (18 pacientes) lesión del tendón de Aquiles en 44.44\%; fútbol Americano (13 pacientes) lesión del LCA en $30.7 \%$, lesión de menisco en $23 \%$, lesión de clavícula en $23 \%$. Al realizar la prueba de $\chi^{2}$ no se reportaron valores estadísticamente significativos.
La cirugía más realizada en hombres fue la artroscopia de rodilla, representando 46 (23\%) del total; plastia del LCA con 36 (18\%); plastia del tendón de Aquiles con 29 (14.5\%); la reducción abierta y fijación interna (RAFI) de clavícula con 12 (6\%), y reducción cerrada y fijación percutánea (RCFP) con 9 (4.5\%). Las cirugías que se realizaron con más frecuencia en el género femenino fueron la artroscopia de rodilla con 15 (42.9\%) casos; la plastia
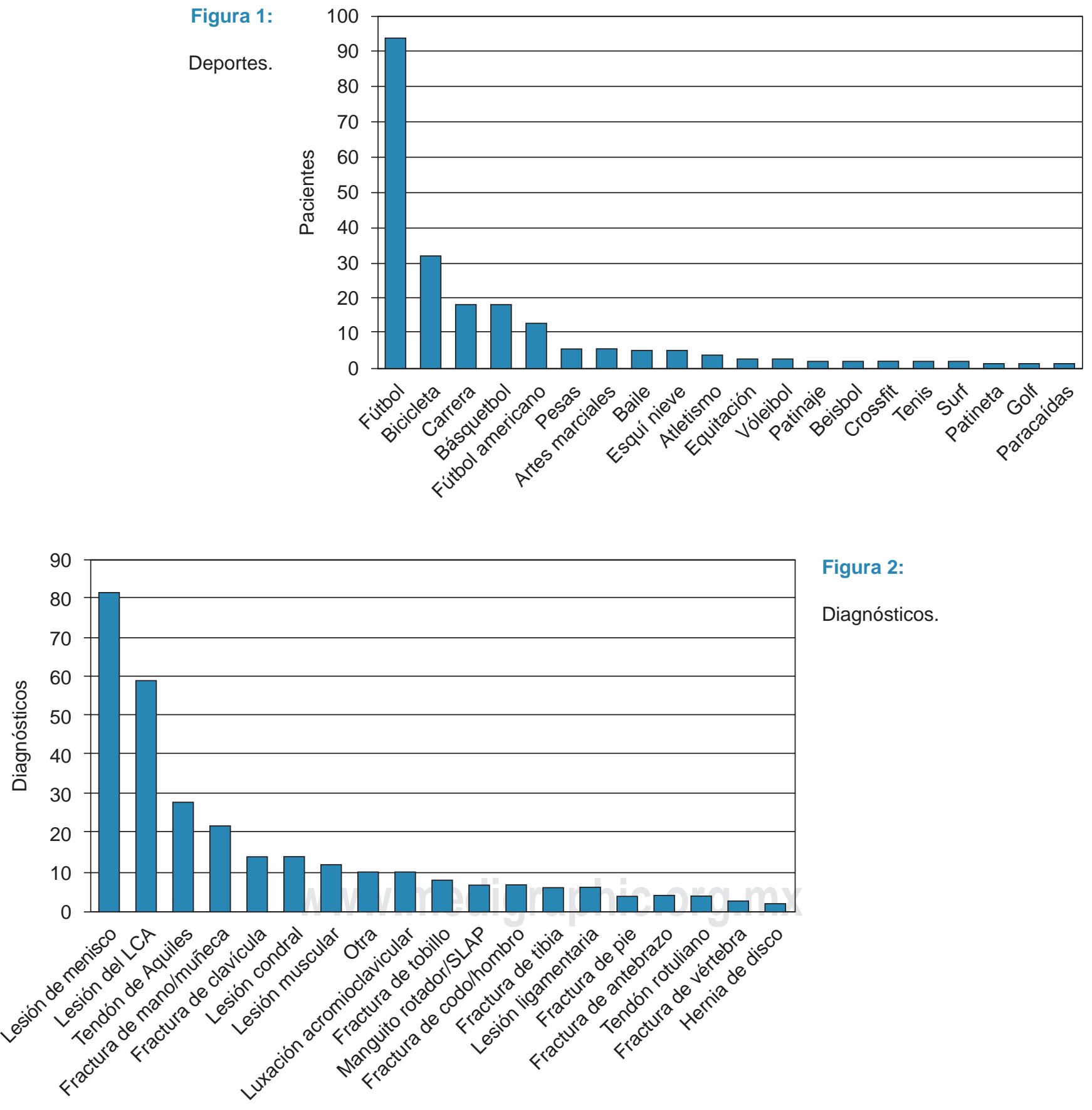

Figura 2:

Diagnósticos. 


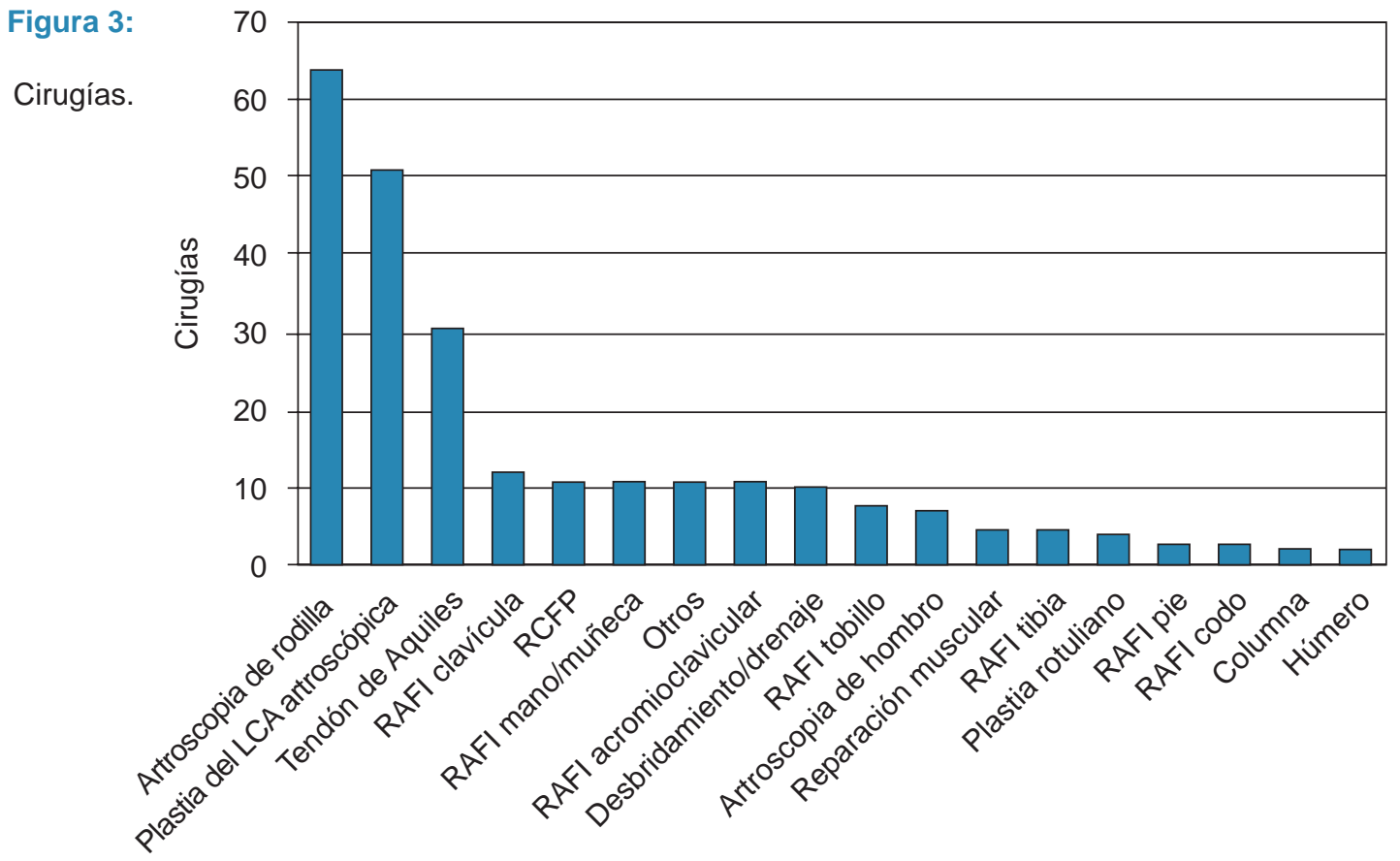

del LCA con 13 (37.1\%); la plastia del tendón de Aquiles con dos (5.7\%); RCFP equivalente a dos (5.7\%) y plastia muscular por igual. No existió significancia estadística en la correlación del tipo de intervención quirúrgica.

La distribución por año se comportó dependiendo el año, se puede observar en la figura 4 según el año y género. Los rangos de edad de los pacientes se reportan en la figura 5.

\section{DISCUSIÓN}

Derivado de la información obtenida en el estudio, se observa que varios datos resultan de utilidad para realizar un análisis detallado. Fue posible comprobar que el fútbol destaca como el deporte más practicado tanto en hombres como en mujeres, representando 39\% de total $y$, por ende, se considera el deporte más popular en México previamente citado por Instituto Belisario Domínguez, ${ }^{4}$ seguido por el básquetbol, vóleibol, caminar y correr. Probablemente, la popularidad del fútbol varía en cada entidad federativa, aunque el tema no fue sujeto a estudio en el presente artículo. De los 93 pacientes que presentan lesión por fútbol -citado por Costa Astur y colaboradores ${ }^{5}$ como el deporte con más lesiones especialmente en países en los que se practica por su gran popularidad- la lesión más común corresponde al menisco con 44 pacientes, seguida de la ruptura del LCA $(n=30)$, fracturas $(n=16)$ y ruptura del tendón de Aquiles $(n=14)$. Hagino y colaboradores ${ }^{6}$ reportaron que aproximadamente $50 \%$ de deportistas activos presenta una lesión meniscal, congruente con el resultado de este estudio. Cabe mencionar que no se toma en cuenta el nivel competitivo del paciente.

El uso de la bicicleta como medio de transporte ha ido adquiriendo mayor relevancia entre la población, particularmente en el caso de la Ciudad de México como alternativa para la movilidad. Del total de los accidentes en bicicleta, sólo $8 \%$ de los pacientes requirieron atención médica, especialmente por lesiones de cráneo. ${ }^{7}$ Asimismo, la clavícula es uno de los sitios más lesionados al practicar este deporte. ${ }^{8}$ De los 32 pacientes con lesión por bicicleta, cinco presentaron fractura de clavícula y cuatro luxaciones acromioclaviculares, sumando así $28 \%$ del total de las lesiones por bicicleta.

Como se hizo mención previamente, el básquetbol es otro de los deportes de mayor popularidad en México por su facilidad y poco costo económico para realizarlo, a lo que se suman factores de tipo cultural y social. El análisis arroja una mayor incidencia de lesiones en hombres que en mujeres. Aquellas que ocurren con mayor regularidad son la lesión del tendón Aquiles, representando siete de 18 casos (38.9\%), por su mecanismo de lesiones de contracción brusca realizados durante la práctica. Sin embargo, en la literatura sobresale que las lesiones más comunes son los esguinces de tobillo y las lesiones del LCA,${ }^{9}$ que equivalen a sólo tres casos de 18 (16.7\%) de ruptura del LCA y ninguna por ruptura ligamentaria de tobillo. 
La artroscopia de rodilla es un procedimiento tanto de diagnóstico como terapéutico que ha tenido grandes avances desde los años 70, específicamente en las patologías meniscales. ${ }^{10}$ Se estima que una tercera parte de las lesiones meniscales se asocian con lesiones deportivas, ${ }^{11}$ en especial en deportes de pivoteo y corte, donde la rodilla es la articulación más afectada. ${ }^{12}$ Por este motivo, la artroscopia de rodilla es el procedimiento más recurrente. A partir de un análisis diferencial por género, se encuentra que, en ambos casos (hombres y mujeres) la cirugía más común es la artroscopia de rodilla, y si se agrega la plastia del LCA, que se realiza vía artroscópica, encontramos que $46 \%$ de todas las cirugías por lesiones deportivas y como etiología más común es el fútbol seguido de las carreras, fútbol americano, básquetbol y baile.

Las fracturas óseas son otras lesiones muy relevantes en los resultados: 63 de 308 (20\%) diagnósticos obedecieron a este tipo de fracturas. Las fracturas óseas relacionadas por actividad física se pueden dividir por estrés repetitivo o traumatismo agudo en evento deportivo. ${ }^{13}$ En los hombres es más frecuente la clavícula y en mujeres las fracturas de mano y muñeca. La etiología deportiva en esta situación fue la bicicleta (35\%), ya que presenta los mecanismos de lesión de más alta energía. El fútbol vuelve a aparecer en esta categoría como la segunda causa (25\%) de fracturas y el fútbol americano en un tercer lugar (9.5\%).

La ruptura del LCA es una de las principales lesiones en personas que realizan deporte. En cuanto a las diferencias entre géneros, según los estudios publicados, en las mujeres predomina la ruptura del LCA en comparación con los hombres. ${ }^{14}$ De acuerdo con los resultados de este estudio, se pudo corroborar esta información: $40 \%$ de las lesiones se presentaron en mujeres y $21 \%$ en hombres. Las lesiones del LCA se encuentran altamente relacionadas con lesiones meniscales entre $26-46 \%{ }^{15}$ En este sentido, 56\% de los pacientes de este estudio a

Figura 4: Género.

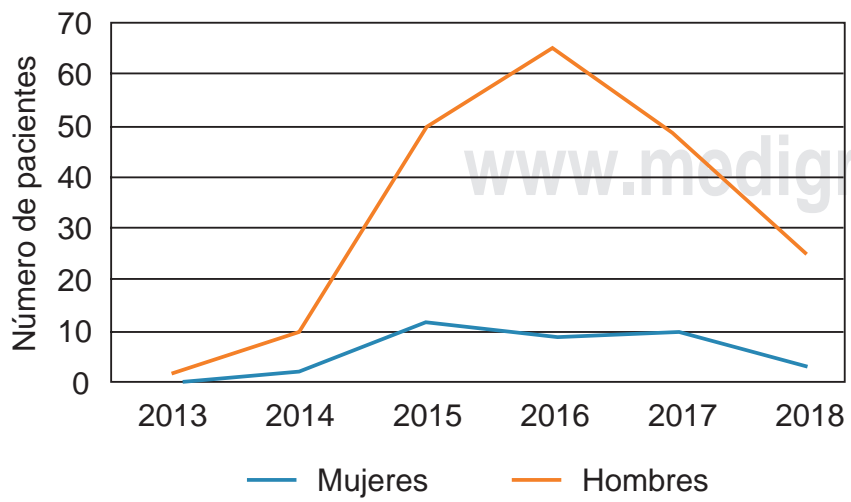

Figura 5: Grupo de edades.

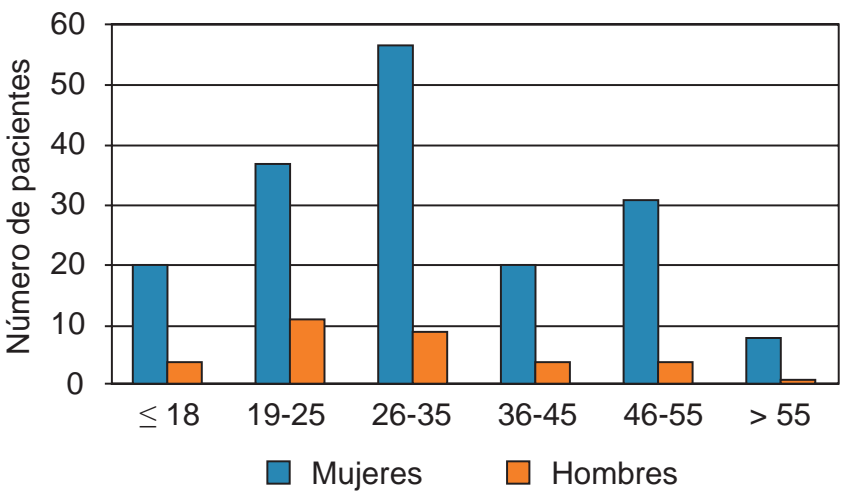

los que se les realizó la plastia del LCA vía artroscópica, adicionalmente presentaban lesión meniscal, siendo el fútbol la causa principal, seguido del fútbol americano (ambos de alta intensidad). El menisco lateral fue el más asociado con lesiones del LCA, dato que no concuerda, ya que el menisco medial se asocia más como lesión concomitante. ${ }^{16,17}$

El presente estudio encuentra limitaciones relacionadas con diversos factores. Por un lado, aun cuando se tomaron en cuenta a los pacientes de 2018, hubiera sido favorable considerar la totalidad del año para ofrecer estadísticas más completas. Por otro lado, el nivel de competitividad no se consideró y, en consecuencia, sería de mayor relevancia incluir este factor en un próximo estudio, así como el momento en el que sucede la lesión, es decir, en una práctica o evento profesional. Por último, los métodos preventivos no se registraron, impidiendo conocer el grado de afectación en los resultados.

\section{CONCLUSIÓN}

El ejercicio y el deporte representan una actividad fundamental en la vida de las personas, por motivos de desarroIlo profesional, cultural, prácticas sociales y, por supuesto, por salud. Las lesiones deportivas por mala práctica, falta de prevención o accidentales pueden ser severas y riesgosas para la salud. Al conocer las lesiones más comunes y deportes a las que se asocian, como médicos especialistas estamos en la posibilidad de brindar la atención necesaria a los pacientes para realizar una prevención exitosa, un diagnóstico más certero y un tratamiento individualizado hacia las personas deportistas, según su nivel competitivo.

\section{REFERENCIAS}

1. Durstine JL, Gordon B, Wang Z, Luo X. Chronic disease and the link to physical activity. J Sport Health Sci. 2013; 2 (1): 3-11. 
2. Walker B. Explicación de la lesión deportiva. La anatomía de las lesiones deportivas. México: Editorial Paidotribo; 2010. pp. 1-2.

3. Fernández FM, Busto VJM. Prevención de lesiones deportivas. Orthotips. 2009; 5 (1): 93-105.

4. Zamora SI. Visor ciudadano No. 59. Prácticas físico-deportivas en México. Instituto Belisario Domínguez. Senado de la República. 2018.

5. Astur DC, Xerez M, Rozas J, Debieux PV, Franciozi CE, Cohen M. Anterior cruciate ligament and meniscal injuries in sports: incidence, time of practice until injury, and limitations caused after trauma. Rev Bras Ortop. 2016; 51 (6): 652-656.

6. Hagino T, Ochiai S, Sato E, Watanabe Y, Senga S. Footballer's lateral meniscus: anterior horn tears of the lateral meniscus with a stable knee. ISRN Surg. 2011; 2011: 170402.

7. Olivier J, Creighton P. Bicycle injuries and helmet use: a systematic review and meta-analysis. Int J Epidemiol. 2017; 46 (1): 278-292.

8. Quillen DM, Wuchner M, Hatch RL. Acute shoulder injuries. Am Fam Physician. 2004; 70 (10): 1947-1954.

9. Taylor JB, Ford KR, Nguyen AD, Terry LN, Hegedus EJ. Prevention of lower extremity injuries in basketball: a systematic review and meta-analysis. Sports Health. 2015; 7 (5): 392-398.
10. Cozcolluela-Cabrejas MR, Sanz-Salanova LA, Jerez JJ, Madrid JM, Romina L, Pérez F. Lesiones producidas en aficionados durante los festejos taurinos populares. Cir Esp. 2019; 97 (1): 34-39.

11. Bach BR Jr, Dennis M, Balin J, Hayden J. Arthroscopic meniscal repair: analysis of treatment failures. J Knee Surg. 2005; 18 (4): 278-284.

12. Manson TT, Cosgarea AJ. Meniscal injuries in active patients. ASM. 2004; 4 (10): 545-552.

13. Kennedy M, Dunne C, Mulcahy B, Molloy M. The sports' clinic: a one year review of new referrals. Ir Med J. 1993; 86 (1): 29-30.

14. DeCoster TA, Stevens MA, Albright JP. Sports fractures. lowa Orthop J. 1994; 14: 81-84.

15. Musahl V, Karlsson J. Anterior cruciate ligament tear. N Engl J Med. 2019; 380 (24): 2341-2348.

16. Borchers JR, Kaeding CC, Pedroza AD, Huston LJ, Spindler KP, Wright RW; MOON Consortium and the MARS Group. Intraarticular findings in primary and revision anterior cruciate ligament reconstruction surgery: a comparison of the MOON and MARS study groups. Am J Sports Med. 2011; 39 (9): 1889-1893.

17. Majewski M, Susanne H, Klaus S. Epidemiology of athletic knee injuries: a 10-year study. Knee. 2006; 13 (3): 184-188. 